\title{
Experimental Study of the Normal Zone Propagation Velocity in Double-layer 2G-HTS wires by Thermal and Electrical Methods
}

\author{
Z. Zhong, H. S. Ruiz, L. Lai, Z. Huang, W. Wang, and T. Coombs
}

\begin{abstract}
The Normal Zone Propagation Velocity (NZPV) of a double-layer second generation (2G) high temperature superconducting (HTS) wire manufactured by American Superconductor has been measured by electrical and thermal methods, and the results have been compared and discussed. The NZPV values determined by the voltage traces are ranging from $3.8 \mathrm{~mm} / \mathrm{s}$ at $0.4 I_{c}$ to $19.2 \mathrm{~mm} / \mathrm{s}$ at $0.9 I_{c}$; while from $5.9 \mathrm{~mm} / \mathrm{s}$ to $18.3 \mathrm{~mm} / \mathrm{s}$ by the temperature traces. NZPV determined by these two approaches agrees well with each other. Also, NZPV of double-layer YBCO tape is close to that of conventional single-layer superconducting tape.
\end{abstract}

Index Terms - Double-layer 2G-HTS tape, normal zone propagation velocity, quench.

\section{INTRODUCTION}

$\mathrm{F}_{\mathrm{a}}$ OR THE DESIGN and implementation of commercial applications such as superconducting fault current limiters and superconducting magnets $[1,2]$ using second generation $(2 \mathrm{G})$ high temperature superconducting (HTS) wires, a comprehensive understanding of the thermal stability of 2G-HTS wires under different conditions of transport current and ambient magnetic fields is required. Furthermore, it is well recognized that one of the major issues involving the use of 2G-HTS wires is the formation of destructive hot spots, or localized thermal instabilities, due to the ineluctable presence of flaws in the microstructure of the superconducting composite. Thus, in order to quantitatively estimate the performance of a $2 \mathrm{G}$ HTS wire, it is important to determine the propagation speed at which the thermal instability travels along the length of a testing sample, known as normal zone propagation velocity (NZPV). Customarily, to assess the NZPV in a $2 \mathrm{G}-\mathrm{HTS}$ wire, the voltage rise at different positions along the sample length are measured. The typical values obtained are in the order of $10^{-3}-10^{-2} \mathrm{~m} / \mathrm{s}[3,4]$ which is two to three orders of magnitude slower than that in low $\mathrm{T}_{\mathrm{c}}$ counterparts [5].

Extensive efforts have been pursued in the past in order to determine the NZPV of different superconductors under

Manuscript received on August 8, 2014. This work was supported in part by the EPSRC under Grant NMZF/064.

Z. Zhong, H. S. Ruiz, Z. Huang, W. Wang, and T. Coombs are with the Engineering Department, University of Cambridge, Cambridge, CB3 OFA, U.K. e-mail: zz272@cam.ac.uk or tac1000@cam.ac.uk.

L. Lai was a visiting student at the Engineering Department, University of Cambridge, Cambridge, CB3 0FA, U.K, and is currently with the Department of Physics, Tsinghua University, Beijing, China.
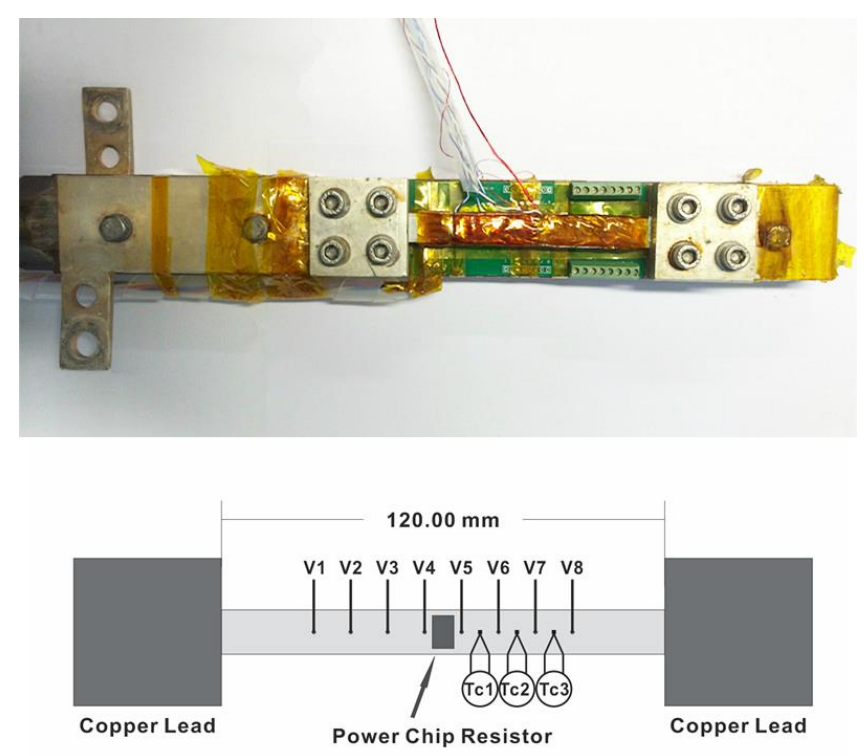

Fig. 1 (Color online) Top: Picture of the mounted sample. Bottom: Schematic illustration of the experimental setup showing the position of 8 voltage taps labelled as V1 to V8, 3 thermocouples labelled as Tc1 to Tc3, and the power chip resistor (heater).

various cryogenic conditions, and typical results are given ranging from $5 \mathrm{~mm} / \mathrm{s}$ to $20 \mathrm{~mm} / \mathrm{s}$ for different applied current [3, 4, 6-9]. However, according to our knowledge, not many studies have been done when superconducting composites are immersed in liquid nitrogen. There are two main benefits for such study, one is that a more homogeneous temperature can be obtained on the entire sample surface, the other is that the effect of Joule heating at current leads can be minimized [10]. This study could help to advance our understanding in voltage development and temperature evolution of resistive type superconducting fault current limiter (SFCL). Also, no measurements of the NZPV for double-layer 2G-HTS wires have been reported in the literature. Thus, in this paper, we present a comparison between the NZPV obtained for the Amperium $^{\circledR}$ stainless steel laminated wire type 8612, when both thermal and electrical methods are taken into consideration. The double YBCO-layer structure and stainless steel sheathing provide high current capacity and robustness which are ideal for superconducting fault current limiters[11]. 
The paper is organized as follows. In Sec. II the experimental setup and procedure pursued for the measurement of the NZPV are presented. In Sec. III the different methods used for the measurement of the NZPV are introduced and the results are explained. Finally, a brief summary of the NZPV values obtained from both methods is given.

\section{EXPERIMENTAL PROCEDURE}

\section{A. Experimental Setup}

The normal zone propagation velocity of Amperium ${ }^{\circledR}$ stainless steel laminated wire type 8612 with double HTS layers has been measured by two different experimental approaches. Experiments were carried out on a sample with $160 \mathrm{~mm}$ length and $12 \mathrm{~mm}$ wide immersed in liquid nitrogen bath.

Once thermal equilibrium had been established, transport current measurements were first performed for self-field condition in order to estimate the critical current across the entire sample. When the standard electric-field criteria of $1 \mu \mathrm{V} / \mathrm{cm}$ is used at $77 \mathrm{~K}$, the measured $I_{\mathrm{c}}$ is $532 \mathrm{~A}$.

In Fig. 1 we show a schematic illustration of the distribution of voltage taps, thermocouples and heater in the aforementioned sample. Eight voltage taps were soldered on the surface of the wire which had been labelled as superconducting side by the manufacturer. The voltage taps with a relative distance between each other of about $10 \mathrm{~mm}$ $( \pm 0.5 \mathrm{~mm})$ were centered over the length of the wire for the simultaneous measurement of the NZPV towards the left and right directions of a small power-chip resistor (51.2 $\Omega$ at room temperature and it has a rather small temperature coefficient from $77 \mathrm{~K}$ to room temperature). This resistor (5.842 $\mathrm{mm}$ width and $8.89 \mathrm{~mm}$ long), also called heater for the sake of simplicity, provides the heat pulse needed for the initialization of the normal zone in the superconducting tape. Concomitantly, towards the right side of the heater and in between the voltage taps, three Type-T thermocouples were glued with thermal compound paste for measuring the time-dependent evolution of the temperature.

During quench there are three thermal paths, i.e., along the superconductor, along the cladding/substrate and into the nitrogen. At $77 \mathrm{~K}$, the $\mathrm{YBCO}$ itself has a thermal conductivity of $17.25 \mathrm{Wm}^{-1} \mathrm{~K}$, this combined with a thickness of 1 micron allows for a heat flux of $1.725 \times 10^{-5}$ watt per degree. The steel is thicker and has a thermal conductivity of $7.9 \mathrm{Wm}^{-1} \mathrm{~K}$ and a thickness of 75 microns, this allows for a heat flux of $59.3 \times 10^{-5}$ watt per degree [12]. The flux into the nitrogen is around 0.3 watt per square centimetre providing temperature difference of 4 degree [13] and this is substantially greater than that in either the YBCO or the steel. Thus heat is conducted away and the quench will not propagate. Therefore, when conducting an experiment with bare tape, voltage rise can only be registered by the two voltage taps closest to the heater. In order to mitigate the thermal leak, we wrapped the superconductor in Kapton ${ }^{\circledR}$ tape $\left(0.21 \mathrm{Wm}^{-1} \mathrm{~K}\right)$ to provide a thermal barrier between the superconductor and surrounding liquid nitrogen bath.

Finally, as shown in Fig. 1, both terminals of the sample were clamped with copper plates with a $\sim 20 \mathrm{~mm}$ overlap. The interval between the current lead and the closest voltage tap is $25 \mathrm{~mm}$ on each side, and the whole setup was immersed in liquid nitrogen bath.

\section{B. Experimental Protocol}

NZPV and MQE measurements were performed at $77 \mathrm{~K}$ in a liquid nitrogen bath. First, the $I_{\mathrm{c}}$ of the sample was measured once the thermal equilibrium had been achieved. The transport current was then slowly ramped up to a certain fraction of $I_{c}$, and kept stable during subsequent measurements. An Agilent 6680A system power supply was applied as the current source. Then, a heat pulse with a fixed width of $200 \mathrm{~ms}$ was generated by a DC power supply (Agilent E3634A). Initially, a relatively small heat pulse was applied, if a quench was not initiated, then the magnitude of the voltage input was increased with a fixed interval of $0.1 \mathrm{~V}$ in order to determine the minimum quench energy (MQE). The current of both instruments was measured by the voltage signals across shunt resistors, i.e., a shunt with rated power of $36 \mathrm{VA}(60 \mathrm{mV}, 600 \mathrm{~A})$ for the current source, and a shunt with rated power of $1 \mathrm{VA}$ $(50 \mathrm{mV}, 20 \mathrm{~A})$ for the heater power supply.

The voltages and thermal potentials over the test sample were recorded simultaneously by a DAQ card (NI PCI-6224) with the speed at up to $250 \mathrm{k}$ data points per second. The cold junctions (reference junctions) of the three thermocouples were all thermally anchored to a bulk copper immersed in a separate liquid nitrogen bath, whose temperature was measured by an additional thermocouple connected to a high precision cryogenic digital thermometer. The entire system was controlled using a LabVIEW platform.

\section{RESULTS AND DISCUSSION}

\section{A. NZPV measurements and quench protection}

In order to propagate the normal zone along the entire conductor, and therefore determine the NZPV, the sample was quenched with a high end-to-end voltage. After establishing the MQE for 2G-HTS wire under various transport current conditions, the test sample was thermally quenched with its corresponding MQE aiming to minimize the effect of the energy input on the accurate estimation of the NZPV.

The NZPV is given by the time $(\Delta t)$ taken for a normal zone front to propagate either from a voltage tap or a thermocouple to the neighbouring one. To define the time period for each of the methods, a reference voltage $\left(\mathrm{V}_{\text {ref }}\right)$ or a reference temperature $\left(T_{\text {ref }}\right)$ is selected in the region where two adjacent voltage or temperature traces are almost parallel to each other. Therefore, the average propagation speed in one specific section can be obtained by direct comparison between the results. The error in $\Delta t$ is estimated 
to be $10 \mathrm{~ms}$, leading to less than 5\% error in NZPV values calculated by both methods.

The time required to absorb the thermal energy during a quench without burning the superconducting tape is much greater than that needed for detecting the voltage rise. Hence, two threshold values were pre-set during the experiments. One threshold value was set to stop the current input when a predefined maximum temperature is reached by the thermocouple Tc1 $(150 \mathrm{~K})$. This protection mechanism is usually activated when a low transport current is given, and in this case a greater heat input is required. The other threshold protects the sample by presetting the maximum voltage of V4-V5 $(0.18 \mathrm{~V})$ (See Fig. 1 ), which is normally exceed when a large current is applied. Nevertheless, due to the high critical current density of the sample, the protection system is usually activated before the quench has propagated along the entire wire. This fact impedes the determination of NZPV by both the voltage taps, and the thermocouples for applied transport current of $I_{\mathrm{t}} / I_{\mathrm{c}} \geq 0.8$ or $I_{\mathrm{t}} / I_{\mathrm{c}} \leq 0.4$. Thus, in order to overcome this difficulty and achieve desired comparison of NZPV determined by electrical and thermal methods, an external magnetic field of $300 \mathrm{mT}$ was applied perpendicular to the sample broad surface, which would substantially reduce the critical current. Once the thermal equilibrium had been established, the critical current under $300 \mathrm{mT}$ external magnetic field was determined, obtaining a final value of $I_{\mathrm{c}}=239 \mathrm{~A}$.

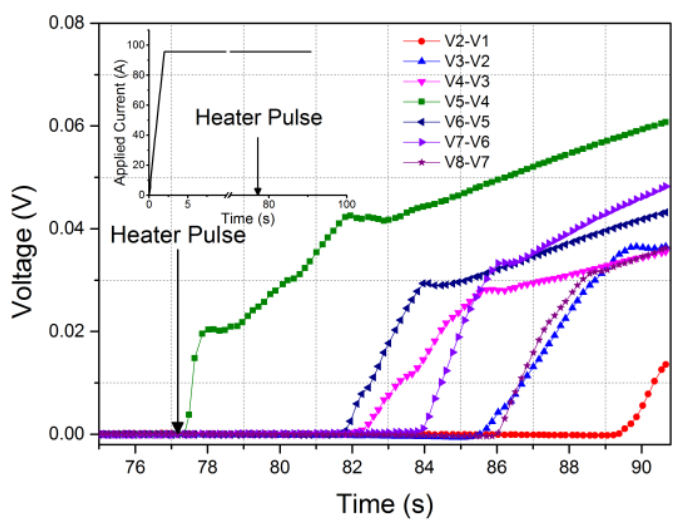

Fig. 2 (Color online) Voltage profiles vs. time measured for the condition of transport current $I_{t} / I_{\mathrm{c}}=0.4$, when the double-layer 2G-HTS wire is subjected to an external magnetic field of $300 \mathrm{mT}$, perpendicular to the surface of the sample. The curves are labelled according to the positions of the voltage taps shown in Fig. 1. Magnitude and duration of the heat pulse are $22 \mathrm{~V}$ and $200 \mathrm{~ms}$, respectively (Arrow indicates the start time of heat pulse).

\section{B. NZPV determined by voltage traces}

The immediate impact of the heat pulse can be observed by the measured voltage rise in the central region of the sample (V5-V4), followed by a rather long time interval for the travelling of the normal zone front to the nearest two sections (See Fig. 2 and Fig. 3). For the voltage differences between $\mathrm{V} 4-\mathrm{V} 3$ and $\mathrm{V} 6-\mathrm{V} 5$ in the case of $I_{\mathrm{t}} / I_{\mathrm{c}}=0.4$, the lag times are $4.8 \mathrm{~s}$ and $4.4 \mathrm{~s}$, respectively. On the other hand, for $I_{\mathrm{t}} / I_{\mathrm{c}}=0.9$, the corresponding lag times are $0.6 \mathrm{~s}$ and $0.7 \mathrm{~s}$, respectively. This fact indicates that according to the voltage profiles, the NZPV increases by an average factor of $7.2 \pm 0.8$ when the applied transport current is increased from $0.4 I_{\mathrm{c}}$ to $0.9 I_{\mathrm{c}}$ as shown in Fig. 4.

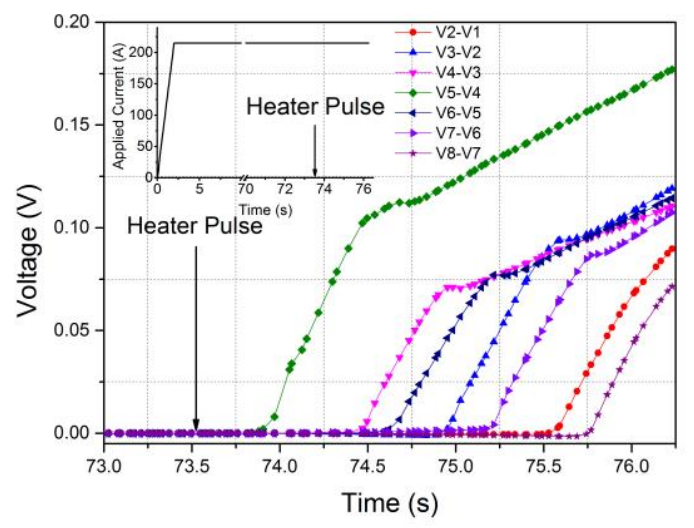

Fig. 3 (Color online) Same as Fig. 2 but with the condition $I_{\mathrm{t}} / I_{\mathrm{c}}=0.9$. Magnitude and duration of the heat pulse are $15.9 \mathrm{~V}$ and $200 \mathrm{~ms}$, respectively (Arrow indicates the start time of heat pulse)..

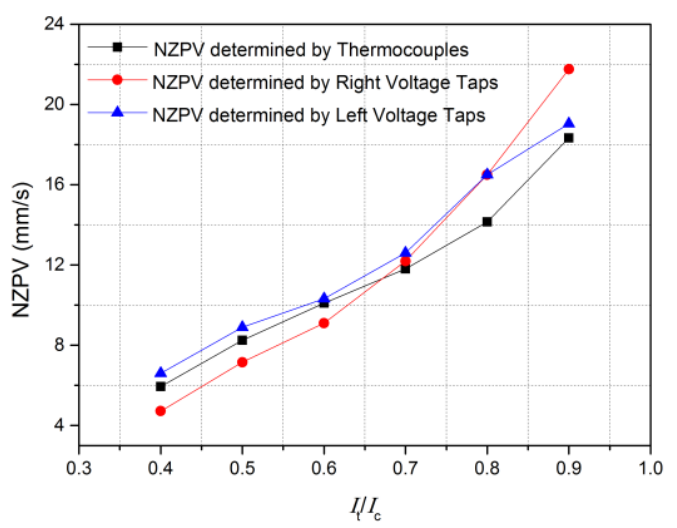

Fig. 4 (Color online) Comparasion of the NZPV determined by the voltage traces and the temperature traces.

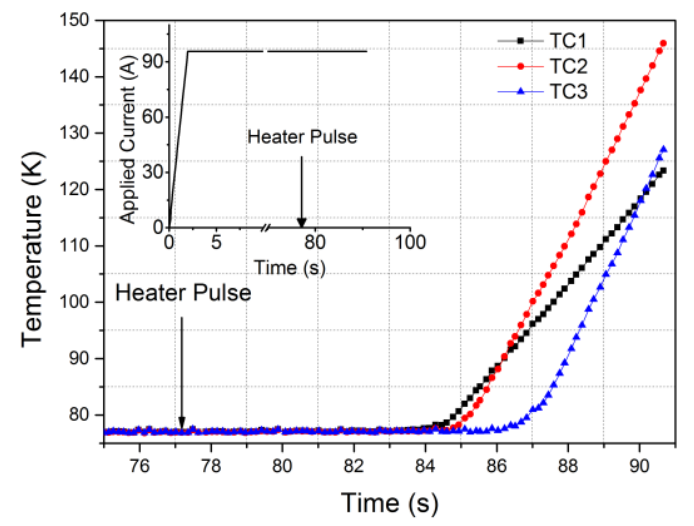

Fig. 5 (Color online) Temperature profiles vs. time measured for the condition of transport current $I_{\mathrm{t}} / I_{\mathrm{c}}=0.4$, when the double-layer 2G-HTS wire is subjected to an external magnetic field of $300 \mathrm{mT}$, perpendicular to the surface of the sample. The curves are labelled according to the positions of the thermocouples shown in Fig. 1. Magnitude and duration of 
the heat pulse are $22 \mathrm{~V}$ and $200 \mathrm{~ms}$, respectively (Arrow indicates the start time of heat pulse).

A reference voltage $\left(\mathrm{V}_{\text {ref }}\right)$ was adopted to estimate NZPV, where $\mathrm{V}_{\text {ref }}=0.01 \mathrm{~V}$ and $\mathrm{V}_{\text {ref }}=0.04 \mathrm{~V}$ were chosen for these two specific cases shown in Fig. 2 and Fig. 3, respectively. It is worth pointing out that the slopes of the left-side voltage traces (V2-V1, V3-V2, and V4-V3) are smaller than those of the right-side voltage traces (V6-V5, V7-V6 and V8-V7) when $I_{\mathrm{t}}=0.4 I_{\mathrm{c}}$. Interestingly, the differences observed between the left and right side voltage traces tend to disappear when the magnitude of the transport current is increased, i.e., when the heat leak is homogenized by the high transport current flowing across the entire sample. In fact, for $I_{\mathrm{t}}=0.9 I_{\mathrm{c}}$, discernible differences are no longer observed (see Fig. 3). For low transport current, the unavoidable inhomogeneity in the boil-off features of the liquid nitrogen over different sections of the sample surface might play a significant role.

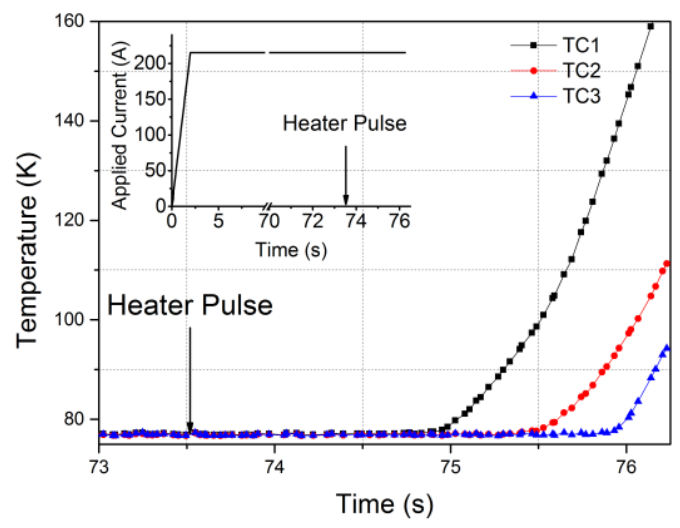

Fig. 6 (Color online) Same as Fig. 4 but with the condition $I_{\mathrm{t}} / I_{\mathrm{c}}=0.9$. Magnitude and duration of the heat pulse are $15.9 \mathrm{~V}$ and $200 \mathrm{~ms}$, respectively (Arrow indicates the start time of heat pulse).

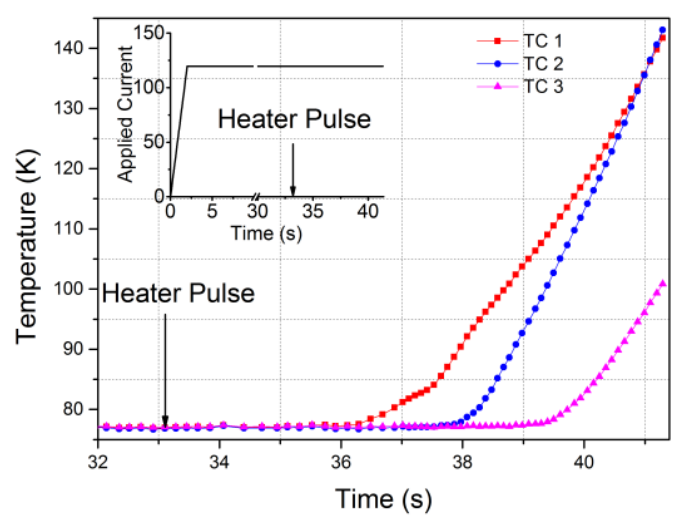

Fig. 7 (Color online) Same as Fig. 4 but in this case no external magnetic field was applied, and the transport current is $I_{\mathrm{t}}=119.5 \mathrm{~A}$. Magnitude and duration of the heat pulse are $19.8 \mathrm{~V}$ and $200 \mathrm{~ms}$, respectively (Arrow indicates the start time of heat pulse

\section{NZPV determined by temperature traces}

Following the above procedure, a similar approach was implemented in order to determine the NZPV by the measurement of the time dependent temperature profiles at different points (see Figs. 5 \& 6). As only parallel temperature traces are eligible to determine the NZPV, Tc2 and $\mathrm{Tc} 3$ were used to determine the NZPV when $I_{\mathrm{t}} / I_{\mathrm{c}}=0.4$, 0.5 , and Tc1 and Tc2 were used to determine the NZPV when $I_{\mathrm{t}} / I_{\mathrm{c}}=0.6,0.7,0.8,0.9$. A reference temperature of $95 \mathrm{~K}$, which is higher than current sharing temperature, was selected for all these cases. In the case of $I_{\mathrm{t}}=0.4 I_{\mathrm{c}}$ and $I_{\mathrm{t}}=$ $0.9 I_{\mathrm{c}}$, the quench propagates from the heater to Tc1 with a lag time of $6.8 \mathrm{~s}$ and $1.1 \mathrm{~s}$, respectively. Therefore, according to the temperature profiles, the NZPV increases by a factor of 6.18 which is slightly smaller than the lowest measured value aforementioned. It worth to mention that, as it is impossible to make ideal thermal contacts between thermocouples and sample, and a certain amount of time is required for thermal diffusion, higher uncertainty may be brought in for NZPV estimation when using temperature traces.

It is to be noticed that for low transport current, the homogeneity of the heat dissipation zone is moderated by the size of the heater and the power of the heat pulse. Thus, under these circumstances the power dissipated by the liquid nitrogen close to the heater may become smaller than in the area further away (See Fig. 5), as the cooling power of liquid nitrogen is not monotonic with temperature [14] Moreover, we have noticed that the distinct signature of this effect, i.e., the crossing between the temperature profiles, is heightened when an external magnetic field is applied to the sample. Basically, this implies that an additional amount of energy needs to be dissipated due to the presence of magnetization currents. Nevertheless, this effect can be observed even for self-field experiments (no external magnetic field is applied) with transport current $I_{\mathrm{t}}=119.5 \mathrm{~A}$ (See Fig. 7). Similar behaviour has been reported for Ag sheathed Bi-2223 superconducting tapes [15] and AMSC Cu-stabilized YBCO coated conductors [16].

\section{CONClusions}

NZPV determined by voltage traces and temperature traces actually represent two different methodologies. The thermocouple is only measuring temperature at one point, while the voltage difference in one section essentially stands for an average temperature over the region. A comparison of NZPV determined by the thermal and electrical methods is presented in Fig. 4. It can be seen that the NZPV increases as the magnitude of the applied transport current increases and NZPV determined by these two approaches agrees well with each other. When comparing the NZPV results with a conventional singlelayer YBCO tape, no significant differences were observed.

\section{REFERENCES}

[1] J. Schwartz, T. Effio, X. Liu, Q. V. Le, A. L. Mbaruku, H. J. Schneider-Muntau, T. Shen, H. Song, U. P. Trociewitz, and X. Wang, "High field superconducting solenoids via high temperature superconductors," Applied Superconductivity, IEEE Transactions on, vol. 18 , pp. 70-81, 2008.

[2] M. Noe and M. Steurer, "High-temperature superconductor fault current limiters: concepts, applications, and development status," Superconductor Science and Technology, vol. 20, p. R15, 2007. 
[3] R. Grabovickic, J. W. Lue, M. J. Gouge, J. A. Demko, and R. C. Duckworth, "Measurements of temperature dependence of the stability and quench propagation of a 20-cm-long RABiTS Y-Ba-Cu-O tape," Applied Superconductivity, IEEE Transactions on, vol. 13, pp. 1726-1730, 2003.

[4] X. Wang, U. Trociewitz, and J. Schwartz, "Near-adiabatic quench experiments on short $\mathrm{YBa} 2 \mathrm{Cu} 3 \mathrm{O} 7-\delta$ coated conductors," Journal of Applied Physics, vol. 101, p. 053904, 2007.

[5] F. Trillaud, F. Ayela, A. Devred, M. Fratini, D. Leboeuf, and P. Tixador, "Quench propagation ignition using single-mode diode laser," Applied Superconductivity, IEEE Transactions on, vol. 15, pp. 3648-3651, 2005.

[6] H. Song and J. Schwartz, "Stability and quench behavior of coated conductor at $4.2 \mathrm{~K}$, self-field," Applied Superconductivity, IEEE Transactions on, vol. 19, pp. 3735-3743, 2009.

[7] X. Wang, A. R. Caruso, M. Breschi, G. Zhang, U. P. Trociewitz, H. W. Weijers, and J. Schwartz, "Normal zone initiation and propagation in Y-Ba-Cu-O coated conductors with $\mathrm{Cu}$ stabilizer," Applied Superconductivity, IEEE Transactions on, vol. 15, pp. 2586-2589, 2005.

[8] F. Trillaud, H. Palanki, U. Trociewitz, S. Thompson, H. Weijers, and J. Schwartz, "Normal zone propagation experiments on HTS composite conductors," Cryogenics, vol. 43, pp. 271-279, 2003.

[9] Y. Iwasa, L. HAIGUN, F. JIARONG, and B. Haid, "Quench and recovery of YBCO tape experimental and simulation results," IEEE Transactions on Applied Superconductivity, vol. 13, pp. 1772-1775, 2003.

[10] Y. Iwasa, J. Jankowski, S.-y. Hahn, H. Lee, J. Bascuñán, J. Reeves, A Knoll, Y. Xie, and V. Selvamanickam, "Stability and quench protection of coated YBCO" Composite" tape," Applied Superconductivity, IEEE Transactions on, vol. 15, pp. 1683-1686, 2005.

[11] www.amsc.com.

[12] http://www.cryogenics.nist.gov/.

[13] R. J. Krane, J. R. Parsons, and A. Bar-Cohen, "Design of a candidate thermal control system for a cryogenically cooled computer," Components, Hybrids, and Manufacturing Technology, IEEE Transactions on, vol. 11, pp. 545-556, 1988.

[14] B. Zeimetz, K. Tadinada, D. Eves, T. Coombs, J. Evetts, and A. Campbell, "Thermal instability and current-voltage scaling in superconducting fault current limiters," Superconductor Science and Technology, vol. 17, p. 657, 2004.

[15] S. Kim, Y. Ueno, A. Ishiyama, H. Okada, S. Nomura, and H. Maeda, "Experiment and numerical analysis of normal zone propagation properties in $\mathrm{Ag}$ sheathed $\mathrm{Bi}-2223$ superconducting tapes," Magnetics, IEEE Transactions on, vol. 32, pp. 2822-2825, 1996.

[16] G. M. Zhang, D. J. Knoll, D. N. Nguyen, P. V. Sastry, X. Wang, and J. Schwartz, "Quench behavior of $\mathrm{YBa} 2 \mathrm{Cu} 3 \mathrm{O} 7$ coated conductor with AC transport current," Applied Superconductivity, IEEE Transactions on, vol. 17, pp. 3874-3879, 2007. 